\title{
Some issues in recent entrepreneurship approaches: joining previous and current theories
}

\author{
Domingo Ribeiro-Soriano $^{1} \cdot$ Juying Zeng $^{2}$ (D)
}

Published online: 10 November 2017

(C) Springer Science+Business Media, LLC, part of Springer Nature 2017

The topic of this special issue is the analysis of theory related to entrepreneurship. Each theory is a lens that allows us to interpret one or other mode of entrepreneurship, to identify a particular set of problems and to seek a variety of solutions to those problems. The special issue is a collection of seven papers.

Business credit is currently the main way to solve the financing constraints of small and medium-sized enterprise. Therefore it is worth studying how to reduce the credit risk of enterprise or to strengthen the risk management ability of enterprise. However, according to the theory of information economics, there exists heavy information asymmetry between banks and enterprise, especially small and medium-sized enterprise, resulting the adverse selection and moral hazard in financing process, which makes the credit risk more difficult to be rated. In this sense, Xu, Yang, and Sun provide literature about index system of micro enterprise credit evaluation and discusses the variables related to enterprise credit. The paper innovatively introduces non parametric method into decision tree based on the preliminary research of logistic regression model and generalized addictive models, too. More specifically, the previous linear and polynomial parts are replaced by non parametric components, and both tree and non parametric relationship among variables are incorporated in order to evaluate enterprise credit more accurately and identify the credit enterprise. Finally, the paper performs a robust test and provides the results are stable. The comprehensive analysis of enterprise credit identification puts forward the suggestion on credit evaluation with the focus of avoiding losses, fostering excellent enterprise, and realizing the optimal allocation of resources.

Domingo Ribeiro-Soriano

domingo.ribeiro@uv.es

Juying Zeng

riverzjy@163.com

1 Universitat de València, Valencia, Spain

2 School of Data Science, Zhejiang University of Finance and Economics, Hangzhou, China 
Kraus, Ribeiro-Soriano and Schüssler concentrate their overview article on a rising method in Entrepreneurship and Innovation research: the fuzzy-set qualitative comparative analysis (fsQCA). Originating in political science and sociology, and based on settheoretical logics, increasing frequency of this novel method particularly in business and management research is being observed. It is characterized through causal asymmetry and well applicability of small sample sizes because causal conditions or their combinations which can lead to an equifinal outcome. The findings reveal that the number of fsQCA applications in these fields has drastically increased during the past few years. Stemming from the systematic literature review, core aspects dealing with firm performance and innovation are identified. Ultimately, the authors provide promising areas with future potential for the application of fsQCA such as the elaboration of the antecedents leading to business model innovation.

There are few articles that have examined the impact of venture capital (VC) on innovation and new business creation. However, either innovation or new business creation is just one aspect of entrepreneurship, the soul of the enterprise. What VC actually affects is entrepreneurship, and the change of innovation and new business creation is a manifestation of the change of entrepreneurship. In this sense, the article wrote by $\mathrm{Gu}$, Qian and Lu proposes a conceptual model that reflects this relation in the context of China, which has an emerging and immature market with high proportion of state-owned capital, imperfect legal system, inexperienced investors, and wrong market orientation. They also pointed out that with two-sided moral hazard problem, it is more complex to see whether VC could promote entrepreneurship in China even though VC does in developed countries. Three influence mechanisms are analized in detail, including capital support, management experience and spillover effect, and a final conclusion is drawn that the total effect of $\mathrm{VC}$ on entrepreneurship is positive in the case of China theoretically. For the further research to identify the real impact of VC on entrepreneurship through the empirical data analysis, the paper suggests eliminating the sample selection bias caused by VC, measuring firm level entrepreneurship by uniform indexes and dividing VC institutions into those with state-owned capital background and those without any state-owned capital background. Also the endogenous problem should be carefully handled since the real effect of VC on entrepreneurship is still in debate due to the possible reverse causality between these two.

As an open and dynamic knowledge-based system, high-tech entrepreneurial enterprises need a large amount of knowledge, information, know-how, intellectual property rights and other innovation resources in the process of its growth. High-tech entrepreneurial enterprises should be able to fully integrate the internal innovation resources and capabilities in the growth process, but also fully obtain and use the valuable innovation resources from external environment of enterprises. In this sense, Pan, Zhang, Song, Ai and Pan analyze the innovation resource integration pattern of hightech entrepreneurial enterprises formed through integration of internal and external innovation resources in the face of demand change and continuous competition. The authors constructs the conceptual framework of high-tech entrepreneurial enterprises' innovation resource integration pattern based on the description of relative theory of high-tech entrepreneurial enterprise and resource integration. This conceptual framework includes two aspects. One is group polymerisation, which is defined as the pattern that the high-tech entrepreneurial enterprise builds innovation network together with peripheral interrelated enterprises, universities and research institutions, government 
departments, financial institutions and other institutions. The other is chain integration, which is defined as the pattern that the high-tech enterprise pay close attention to the supplier, the client, the upstream and downstream enterprises. On basis of it, the authors concretely analyze the peripheral structure of group polymerisation and chain integration in high-tech entrepreneurial enterprises. This study provides enlightenment for high-tech entrepreneurial enterprises to maintain a sustainable competitive advantage in the growth process.

Liguori, Bendickson, and McDowell seek to connect social cognitive career theory to the entrepreneurial intentions and outcome of an entrepreneur. This theory attempts to examine the multiple factors, both cognitive and contextual, that exist between people and their careers. By applying this theory to the field of entrepreneurship, the authors offer insights into an entrepreneur's development and motivation. They theorize that the two inputs proposed by this theory, person inputs, which includes items such as generalized self-efficacy, gender, and minority status, and environmental or background inputs such as prior work experience, prior entrepreneurship experience and prior family business exposure, will have a strong impact on the entrepreneurial self-efficacy and the entrepreneurial outcome expectations. While some previous literature shows mixed results in applying person-centered inputs to entrepreneurial intentions, the authors express through the use of the social cognitive career theory approach, that researchers should, when possible, distinguish between generalized and entrepreneurial self-efficacy in order to more clearly define the links to entrepreneurial outcomes expectations.

Zeng establishes a theoretically framework to understand the fostering path of ecological sustainable entrepreneurship within big data internet system (specifically within the comprehension in the situation in developing context). Under the fostering path of traditional entrepreneurship, enterprises normally pursues internal economic profit first, traditional growth modes has encountered with frustrating social and environmental problems, while the intervention and governance of controlling or alleviating the negative externalities of enterprises do not bring about satisfying results since the traditional entrepreneurs do not take social, environmental and sustainable responsibilities as their imperative responsibility. In this sense, he provides firstly provides reviewing of existing literatures concerning traditional entrepreneurship, social entrepreneurship, sustainable and environmental entrepreneurship, and finds the positive impact of big data internet system has not included into the topics. Secondly, the research concretely analyzes the emerging public welfare enterprises within big data internet system and shows that the ecological sustainable entrepreneurship has been fostered with positive ecological or environmental, social, and sustainable concern as their primary task during their whole operation period. Most importantly, the research provides the identification of fostering path of ecological sustainable entrepreneurship within big data internet system, and indicates the fostering path lies in the dimensions of (1) positive ecological or environmental, social, and sustainable concern; (2) collective spirit, challenge and learning; (3) crowd funding in financial start-up capital; and (4) shorten growth period and even greater economic profit than traditional enterprises.

Sharing economy is a long applicable concept, which allows the under-utilized resources to be shared and with affordable expenses. On the other hand, long-term care is a serious problem, exhausting various resources. The application of sharing 
economy can help to facilitate long-term care in various aspects. Huarng renders propositions and a research framework for the application and points out many business opportunities for entrepreneurs. Particularly, entrepreneurship in high-tech and innovation can definitely find positions in the application. 